Mens

Revue d'histoire intellectuelle et culturelle

mens

\title{
Le magazine canadien-français : un média américain ?
}

\section{Denis Saint-Jacques et Marie-José des Rivières}

Volume 12, numéro 2, printemps 2012

Enjeux et modalités des transferts culturels dans la vie artistique canadienne-française de la première moitié du $\mathrm{XX}^{\mathrm{e}}$ siècle

URI : https://id.erudit.org/iderudit/1013872ar

DOI : https://doi.org/10.7202/1013872ar

Aller au sommaire du numéro

Éditeur(s)

Centre de recherche en civilisation canadienne-française

ISSN

1492-8647 (imprimé)

1927-9299 (numérique)

Découvrir la revue

Citer cet article

Saint-Jacques, D. \& des Rivières, M.-J. (2012). Le magazine canadien-français : un média américain ? Mens, 12(2), 17-36. https://doi.org/10.7202/1013872ar

\section{Résumé de l'article}

L'article examine la qualification d'» américain » donnée au magazine canadien-français du premier tiers du $\mathrm{xx}^{\mathrm{e}}$ siècle. Si l'opinion des contemporains et la présentation matérielle apparaissent comme des indices en ce sens, une comparaison avec les formes dominantes des magazines français et américains de la même époque révèle aussi un critère " d'esprit " qui confirmerait la justesse de cette appréciation. Pourtant, un autre aspect fondamental, le contenu de fiction, s'affirme absolument français et conduit à réévaluer l’américanité de ce média. 


\title{
Le magazine canadien-français : un média américain ${ }^{1}$ ?
}

\author{
Denis Saint-Jacques et Marie-José des Rivières \\ Département des littératures \\ Centre de recherche interuniversitaire sur la littérature \\ et la culture québécoises de l'Université Laval
}

\section{Résumé}

Larticle examine la qualification d' " américain » donnée au magazine canadien-français du premier tiers du $\mathrm{xx}^{\mathrm{e}}$ siècle. Si l'opinion des contemporains et la présentation matérielle apparaissent comme des indices en ce sens, une comparaison avec les formes dominantes des magazines français et américains de la même époque révèle aussi un critère « d'esprit » qui confirmerait la justesse de cette appréciation. Pourtant, un autre aspect fondamental, le contenu de fiction, s'affirme absolument français et conduit à réévaluer l'américanité de ce média.

\section{Abstract}

This article examines the adjective "American" associated to French Canadian magazines of the first third of the twentieth century. The material

\footnotetext{
${ }^{1}$ Cet article constitue la synthèse de deux communications remaniées. La première, écrite par les deux mêmes auteurs, a été publiée sous le titre « Le magazine en France, aux États-Unis et au Québec », dans Jacques Migozzi et Philippe Le Guern (dir.), Productions(s) du populaire : colloque international de Limoges (2002), Limoges, PULIM, 2005, p. 29-37; l'autre a fait l'objet d'une conférence à la journée d'études Enjeux et modalités des transferts culturels dans la vie artistique québécoise de la fin du $X I X^{e}$ siècle au milieu du XX siècle, organisée par Nova Doyon et Stéphanie Danaux à l'Université de Montréal, le 25 mai 2010.
}

Mens, vol. XII, nº 2 (printemps 2012) 
presentation of those magazines and the opinion of the contemporaries suggest that the association is correct. A comparison with French and American magazines of the same period also indicates a connection at the "spiritual" level. However, another aspect of those French Canadian publications, their content in terms of fiction, gives them a resolutely French aspect and allows us to put in perspective their "américanite".

Ces dernières années, plusieurs travaux ont permis de mieux éclairer la contribution du magazine moderne aux transformations du paysage médiatique des $\mathrm{XIX}^{\mathrm{e}}$ et $\mathrm{XX}^{\mathrm{e}}$ siècles. L'historiographie rend compte des magazines selon deux aspects principaux, soit selon un point de vue historique et selon une perspective sociologique, celle des Cultural Studies². Du côté des études culturelles, deux ouvrages nous servent de cadre conceptuel pour cet article : tout d'abord, Selling Culture: Magazines, Markets, and Class at the Turn of the Century, où Richard M. Ohmann considère le magazine comme une tentative achevée de constituer une idéologie nationale commune aux États-Unis. Le magazine trouve comme public, au début du $\mathrm{xx}^{\mathrm{e}}$ siècle, la nouvelle classe moyenne, celle de l'American Dream. Ensuite, Magazines for the millions: Gender and Commerce in the Ladies' Home Journal; and the Saturday Evening Post, 1880-1910, dans lequel Helen Damon-Moore approfondit le lien entre la féminité et le commerce, un lien particulièrement rentable pour les producteurs de magazines féminins et les annonceurs ${ }^{3}$.

${ }^{2}$ Les Cultural Studies s'intéressent particulièrement aux magazines britanniques et américains, mais ne s'y limitent pas. Voir, par exemple, le collectif international qui adopte cette approche pour analyser les magazines de nombreux pays, Annabelle Cone et Dawn Marly (dir.), The Francophone magazine Inside and Outside France, New Orleans, University Press of The South, 2010.

3 Richard M. Ohmann, Selling Culture: Magazines, Markets, and Class at the Turn of the Century, London, New York, Verso, 1996; Helen Damon-Moore, Magazines for the millions: Gender and Commerce in the Ladies' Home Journal; and the Saturday Evening Post, 1880-1910, Albany, State University of New York Press, 1994. 
Si l'on dispose de vues d'ensemble pour des pôles d'influence comme les États-Unis, mais aussi pour la France ${ }^{4}$, ou encore pour le Canada anglais ${ }^{5}$, force est de constater le peu d'intérêt suscité en ce domaine par le cas du Canada français et plus strictement du Québec, pour lequel il n'existe pas encore une histoire générale de la presse $e^{6}$. On peut supposer que ce manque de curiosité tient à ce que le magazine canadien-français ne s'impose vraiment qu'à partir du deuxième tiers du XIx ${ }^{e}$ siècle et qu'à l'époque il apparaît comme un avatar parmi d'autres des produits qui diffusent la culture américaine au Canada.

C'est ainsi que le présentent à l'époque les gens du métier. En 1907, les publications Poirier, Bessette et Cie lancent à Montréal La Revue populaire, conçue expressément dans le but de «soutenir [...] la comparaison avec tout autre magazine américain de même prix ${ }^{7}$ ». On notera la formule " tout autre magazine américain ». On trouve un peu plus tard, en 1927, dans un roman cette fois, Les sacrifiés d'Olivier Carignan, une remarque dans le même esprit : « Au moins, quand je suis parti, il y avait une couple de revues à Montréal dans lesquelles il ne m'aurait pas déplu d'écrire. Mais maintenant, il ne

${ }^{4}$ Gilles Feyel, « Naissance, constitution et épanouissement d'un genre de presse aux limites floues : le magazine ", Réseaux, sous la direction de Jean-Marie Charon et Rémy Rieffel, "La presse magazine ", vol. xix, no 105 (2001), p. 19-52.

5 Fraser Sutherland, The Monthly Epic: A History of Canadian magazines (17891989), Toronto, Fitzhenry \& Whiteside, 1989; Mary Vipond, The Mass Media in Canada, Toronto, James Lorimer \& Company, 2000.

${ }^{6}$ Voir André Beaulieu, Jean Hamelin et al., La presse québécoise des origines à nos jours, Québec, Les Presses de l'Université Laval, t. I à VII, 1973-1990 ; Denis SaintJacques, "Les magazines populaires en français ", dans Yvan Lamonde, Patricia Fleming et Fiona A. Black (dir.), Histoire du livre et de l'imprimé au Canada, vol. II : 1840-1918, Montréal, Presses de l'Université de Montréal, 2005, p. 333-337; Marie-José des Rivières, Denis Saint-Jacques et Carole Gerson, "Les magazines féminins ", dans Carole Gerson et Jacques Michon (dir.), Histoire du livre et de l'imprimé au Canada, vol. III : 1918-1980, Montréal, Presses de l'Université de Montréal; Toronto, University of Toronto Press, 2007, p. 263-267; et Marie-José des Rivières, Châtelaine et la littérature, 1960-1975, Montréal, L'Hexagone, 1992. 7 La Revue populaire, Montréal, Cie de Publication Alpha, vol. 1, nº 1 (décembre, 1907). 
reste plus rien. Les revues sont des magazines américains... ${ }^{8}$ " Enfin, dans une enquête de La Revue dominicaine consacrée à "Notre américanisation ", Jean Bruchési déclare : " notre peuple achète et lit d'abord des magazines américains " et ajoute : "nous ne possédons aucun grand magazine canadien-français dont le fond et la tenue reflètent véritablement un esprit français ${ }^{9}$. " Or Bruchési paraît bien autorisé à parler ainsi, car il a occupé la fonction de rédacteur en chef à La Revue moderne de 1930 à 1936, il est professeur à l'École des sciences sociales, économiques et politiques de l'Université de Montréal, et Maurice Duplessis le choisit pour devenir sous-secrétaire de la province de Québec en 1937, l'équivalent d'un poste de sousministre aux Affaires culturelles aujourd'hui. De la sorte, propriétaires, auteurs et directeurs de publication s'entendent pour reconnaître ce qui leur paraît une évidence : le magazine canadien-français est un média américain.

Faut-il le croire? Ces magazines ne sont-ils pas écrits en français, produits au pays et rédigés en bonne part par des journalistes d'ici? Pour les contemporains - tant propagateurs, éditeurs, que détracteurs comme Bruchési ou Carignan -, il semble bien qu'il ne faille pas s'en tenir à une vue si superficielle. C'est "l'esprit ", nous dit Bruchési, qui détermine l'identité d'un peuple, et sans doute, les éditeurs Poirier et Bessette sont-ils d'accord avec lui sur ce point. Cette appréciation ne peut se comprendre qu'en restituant le contexte qui la fonde. Il est nécessaire de revenir sur l'histoire du média pour l'expliquer. Nous comparerons d'abord son développement en Angleterre, en France et aux États-Unis où sont mis au point les principaux modèles qui influencent les éditeurs du Canada français. Puis, nous prendrons en compte les premières revues destinées au grand public canadienfrançais au XIX ${ }^{\mathrm{e}}$ siècle, qui ne sont pas encore identifiées à leur époque

${ }^{8}$ Olivier Carignan, Les sacrifiés, Montréal, Éditions du Mercure, 1927, p. 109.

9 Cité dans Marie-José des Rivières et Denis Saint-Jacques, " Notre américanisation ", dans Yvan Lamonde et Denis Saint-Jacques (dir.), 1937 : un tournant culturel, Québec, Les Presses de l’Université Laval, 2009, p. 154. 
comme magazines. Nous retiendrons ensuite comme exemples représentatifs de la première moitié du $\mathrm{xx}^{\mathrm{e}}$ siècle, La Revue populaire et La Revue moderne, périodiques à forte longévité et à forte circulation ${ }^{10}$ dont se rapproche Le Bulletin des agriculteurs durant les années 1940 et 1950 , période où sa circulation domine celle de tous les autres ${ }^{11}$. Nous n'examinerons pas le cas du magazine depuis 1960 , avec, par exemple, Châtelaine ou L'Actualité, qu'on a coutume, par commodité et indépendamment de leur ligne éditoriale, de désigner plutôt comme " québécois " même si dès leurs débuts ils étaient publiés par une entreprise torontoise, Maclean-Hunter.

\section{L'évolution du magazine en Angleterre et en France}

Les chercheurs français se sont peu intéressés au cas du magazine. L'ouvrage de Jean-Pierre Rioux et Jean-François Sirinelli sur La culture de masse en France : de la Belle Époque à aujourd'hui laisse voir que les choses sont en train de changer. Si, dans son chapitre sur "L'horizon américain ", Jacques Portes ne dit rien du magazine, Jean-Yves Mollier consacre une section à la «[p]resse spécialisée et [au] lectorat de masse " à la Belle Époque, que Christian Delporte prolonge et complète dans son chapitre "Au miroir des médias ${ }^{12}$ ". Mais c'est Gilles Feyel qui fournit la synthèse la plus achevée à ce jour dans un article de la revue Réseaux dont nous suivrons ici brièvement le développement en ce qui concerne le magazine anglais et français ${ }^{13}$.

${ }^{10}$ La Revue populaire (1907-1963) atteint un tirage de 127363 en 1963 (voir Beaulieu, Hamelin et al., La presse québécoise des origines à nos jours, t. IV : 18961910, 1979, p. 266-269; La Revue moderne (1919-1960) atteint un tirage de 101650 en 1960 (voir Beaulieu, Hamelin et al., La presse québécoise des origines à nos jours, t. V: 1911-1919, 1982, p. 294-295.

11 Son tirage est de 145000 en 1948 (voir Beaulieu, Hamelin et al., La presse québécoise des origines à nos jours, t. IV : 1896-1910, p. 171.

12 Jean-Pierre Rioux et Jean-François Sirinelli (dir.), La culture de masse en France : de la Belle Époque à aujourd'hui, Paris, Fayard, 2002.

13 Feyel, "Naissance, constitution et épanouissement d'un genre de presse aux limites floues : le magazine». 
On situe habituellement le début de l'aventure des magazines en Angleterre en 1731 avec la parution du Gentleman's Magazine, première revue à porter ce nom qui renvoie à " magasin " français, avec l'idée d'une diversité des rubriques correspondant à celle qu'offre le magasin, ce qui lui confère une connotation commerciale qui ne se perdra pas. Dès lors, le magazine est et restera un périodique comportant des articles accessibles sur des thèmes diversifiés. La France y vient avec un certain retard à la fin du siècle avec le Magasin encyclopédique qui n'apparaît qu'en 1792. La transformation suivante se produit à nouveau en Angleterre quand le magazine lie sa fortune à l'illustration afin de toucher un public plus large. Le Penny Magazine, fondé en 1832, lance le mouvement, vite suivi un peu partout en Occident. Il ne faut pas se faire d'illusion sur l'élargissement du public en France, l'image coûte cher : ni le Musée des familles, ni L'Illustration, ni Le Monde illustré ne concurrencent vraiment les journaux populaires, dont le succès dépend d'abord du roman-feuilleton. Car l'abonnement annuel au magazine illustré reste relativement coûteux. Ces revues illustrées ne portent pas, de fait, le nom de «magazine » qui, au XIX ${ }^{\mathrm{e}}$ siècle, apparaît comme un terme anglais, pour lequel il n'existe pas d'entrée dans le Dictionnaire de la langue française d'Émile Littré ${ }^{14}$.

L'illustration était d'abord passée par la gravure sur bois en 1832, avant qu'on utilise la zincographie à partir du milieu du siècle, mais le progrès décisif en ce sens résulte de l'arrivée de la photographie en similigravure à la fin du siècle. La Vie illustrée, qui naît en 1898, y a recours et elle est aussitôt largement suivie par d'autres magazines dans cette démarche. Feyel affirme : "C'est manifestement des premières années de ce siècle [le $\left.\mathrm{xx}^{\mathrm{e}}\right]$ qu'il faut dater la presse magazine d'aujourd'hui ${ }^{15}$. " Il note que ces nouvelles « revues " qui se convertissent bientôt à l'héliogravure refusent de se considérer comme des " magazines "; il soutient pourtant qu'elles en sont, "voire des

14 Émile Littré, Dictionnaire de la langue française, Paris, Hachette, 1873-1874, vol. III, p. 374.

15 Feyel, "Naissance, constitution et épanouissement d'un genre de presse aux limites floues : le magazine ", p. 28. 
magazines de luxe ${ }^{16}$ ", précise-t-il. Cette réserve à avouer ses dettes doit au moins être rapportée à la flamboyance avec laquelle s'affiche au même moment la grande révolution du magazine aux États-Unis. Les pratiques auxquelles la France emprunte viennent d'ailleurs, mais la discrétion de ses éditeurs à ce propos indique que cet emprunt ne se réalise pas sans une certaine retenue, qui concerne l'aspect commercial. Les contenus variés, l'illustration et même le bas prix de vente, tout semble comparable, sauf la publicité. En France, celleci se trouve cantonnée à des pages en début et en fin de numéro et ne dépasse pas le quart de l'espace total. Sa fonction parait accessoire alors qu'aux États-Unis elle envahit l'ensemble des pages imprimées.

Durant l'entre-deux-guerres, ces revues proliferent et fragmentent de plus en plus leurs publics. Le magazine américain Vogue connait une édition française dès 1921 : il faut bien apprécier cette percée dans un pays comme la France qui croit alors régenter la mode internationale. $V u$ impose, à partir de 1928, une nouvelle formule où la photo prime sur le texte. Les Américains sauront s'en servir pour créer Life en 1936. Mais la transformation décisive survient en fin de compte quand, avec Marie-Claire en 1937 et Match en 1938, " la publicité sort de son isolement pour envahir [...] tout le corps $\mathrm{du}[\ldots]$ magazine $^{17}$ ». Ce sont déjà des magazines au sens où nous l'entendons aujourd'hui : "de véritables espaces publicitaires ${ }^{18}$ ». La formule s'impose et restera acquise. Ainsi, le magazine français, malgré quelques apports spécifiques, reste redevable à ses débuts à des impulsions venues d'abord de Grande-Bretagne, puis, au Xx ${ }^{\mathrm{e}}$ siècle, des États-Unis.

\section{L'essor du magazine aux États-Unis}

S'il est facile de s'accorder avec Feyel quand il affirme que le magazine en arrive en France à « jouir à la fin du xx siècle d'une souveraineté

\footnotetext{
16 Ibid., p. 29.

17 Ibid., p. 37.

18 Ibid., p. 37.
} 
quasi absolue 19 ", on constate que cette domination médiatique se produit beaucoup plus tôt en Amérique du Nord où les tirages des chefs de file dans le domaine dépassent déjà le million d'exemplaires au tournant $\mathrm{du} \mathrm{Xx}^{\mathrm{e}}$ siècle, quand la population américaine franchit la barre des 75 millions. C'est justement ce tournant du siècle que les ouvrages de Damon-Moore et d'Ohmann choisissent de mettre en valeur pour y situer l'apparition de la forme moderne du magazine américain $^{20}$.

Aux États-Unis, la grande aventure de l'expansion territoriale du XIX ${ }^{\mathrm{e}}$ siècle donne à cette nation un immense espace qui offre aux médias une tâche d'homogénéisation nationale où les nécessités du commerce convergent avec l'idéologie de construction identitaire. À la fin du XIX ${ }^{\mathrm{e}}$ siècle, la géographie physique étendue du pays crée une décentralisation progressive. Celle-ci ajoute aux grandes villes, situées et fondées dans le Nord-Est durant les premiers siècles de la colonisation (Boston, New York et Philadelphie), de nouveaux pôles économiques plus éloignés. Il s'agit d'abord de Pittsburgh, ville de l'acier, puis de Chicago, port intérieur et ville des abattoirs, avant que ce ne soit Los Angeles, tête du chemin de fer transcontinental et grand port du Pacifique. Au même moment, éclate la troisième révolution industrielle, qui stimule la fabrication standardisée des produits de consommation domestique et qui fait des États-Unis la plus puissante économie du monde. De grandes usines se mettent à livrer à bas coût, depuis ces pôles économiques diversifiés, les biens domestiques d'usage courant, alimentaires, de toilette ou d'entretien, que les ménages obtenaient auparavant de boutiquiers artisans qui leur étaient voisins. Si le chemin de fer assure l'expédition à distance de ces nouveaux produits, une publicité spécifique portant sur ces articles est nécessaire pour renseigner les consommateurs souvent éloignés des lieux de production. Les médias « traditionnels » semblent

19 Ibid., p. 21.

20 Ohmann, Selling Culture: Magazines, Markets, and Class at the Turn of the Century; Damon-Moore, Magazines for the millions: Gender and Commerce in the Ladies' Home Journal; and the Saturday Evening Post, 1880-1910. 
mal s’y prêter. La décentralisation évoquée plus haut touche aussi la presse quotidienne : il n'y a pas de journal américain couvrant efficacement l'ensemble du pays. Il n'y en a, à vrai dire, toujours pas aujourd'hui. Pour que les nouvelles marques de commerce réussissent la conquête du grand marché national, il reste à créer une forme médiatique tout aussi nationale.

Le magazine, revue populaire illustrée à fort contenu publicitaire, investit le créneau disponible et prolifere avec une rapidité qui confirme l'existence d'un marché pour ce type d'imprimé. De 1860 à 1900, les magazines progressent de 280 à 1800 titres. Notons les noms de quelques nouveaux venus qui connaittront une longue fortune : McCall's, Good Housekeeping, Cosmopolitan, National Geographic, Vogue. En 1900, plus de 50 magazines nationaux se trouvent solidement établis avec une circulation dépassant pour chacun les 100000 exemplaires.

Deux facteurs réorganisent les publics cibles : le premier concerne la montée de la nouvelle classe moyenne, qui résulte de l'expansion soutenue de l'économie durant le dernier tiers du XIX $x^{e}$ siècle. Le magazine américain y reconnaît sa clientèle idéale et convainc le grand public que cette classe représente l'avenir collectif du peuple américain. Il joue ainsi efficacement son rôle d'homogénéisation nationale. Le second facteur touche à la reconnaissance du nouveau pouvoir de la femme comme consommatrice. Comme la maitresse de maison gère les achats domestiques, la publicité se doit de la viser directement et, plus largement, le magazine se doit de s'ajuster à elle, même dans le cas où il prétend s'adresser à une audience familiale. Justement, un périodique domine tous les autres en 1900, dépassant le million d'exemplaires par numéro, le Ladies' Home Journal. La description qu'on peut en faire n'étonnerait pas beaucoup les lectrices un siècle plus tard. Publicité, mode, cuisine, santé, psychologie pratique, questions d'intérêt social, voilà une formule qui vaut encore aujourd'hui. Ajoutons-y littérature et musique en feuilles, rubriques qui se sont effacées au cours des temps. Reste, au-delà des contenus, à décrire matériellement le support : grand format, illustration en 
couleurs le plus souvent pleine page en couverture sur papier glacé, illustrations et publicités abondantes insérées dans le corps du texte. Cela suffit à distinguer ces périodiques des revues plus intellectuelles ou religieuses et à caractériser leur souci de toucher un public large grâce à une grande facilité de lecture. En somme, un support matériel spécifique, ainsi que la recherche et la constitution d'un public national fondées sur la publicité, enfin une orientation visant les femmes comme consommatrices constituent les caractéristiques fondamentales du « magazine américain » du tournant du $\mathrm{xx}^{\mathrm{e}}$ siècle.

\section{Le développement du magazine au Québec}

Il existe au Québec, à partir du milieu du XIX ${ }^{e}$ siècle, des périodiques qui introduisent progressivement dans la presse d'opinion de nouvelles rubriques dans un but de divertissement ${ }^{21}$. Par exemple, Étienne Parent crée en 1840 un supplément hebdomadaire au journal Le Canadien, "Le coin du feu »; il y donne surtout du roman et de la nouvelle, venus de France. Ce supplément disparaît dès 1841. Puis, Le Ménestrel (1844-1845) ajoute à la littérature "légère " des partitions musicales, toujours d'" origine française ». Une nouvelle tentative, l'Album littéraire et musical de la Revue canadienne fait également long feu. Ces publications visent un public bourgeois et ouvrent une voie qui paraît alors toute française.

La Gazette des campagnes (Kamouraska, 1861), sous-titrée Journal illustré d'enseignement pratique et populaire d'agriculture et de colonisation $^{22}$, se tourne vers la classe sociale la plus nombreuse au Canada français et tiendra plus de trente ans. L'illustration et la publicité (deux pages sur huit) en font un ancêtre du magazine au

${ }^{21}$ Voir notamment Kenneth Landry, «La diffusion de la littérature au Québec vers le milieu du XIX ${ }^{e}$ siècle : le rôle des recueils littéraires (miscellanées et albums) ", dans Aurélien Boivin, Gilles Dorion et Kenneth Landry (dir.), Questions d'histoire littéraire : mélanges offerts à Maurice Lemire, Québec, Nuit blanche éditeur, 1996, p. 45-59.

22 Voir Pierre Galipeau, "La Gazette des campagnes ", dans Fernand Dumont, JeanPaul Montminy et Jean Hamelin (dir.), Idéologies au Canada français :1850-1971, Québec, Les Presses de l'Université Laval, 1971, p. 149-178. 
Canada français ${ }^{23}$. En 1869, à Montréal, le Canadian Illustrated News, premier journal illustré à la leggotypie au Canada donne modèle à L'Opinion publique (Montréal, 1870), dirigée d'abord par LaurentOlivier David ${ }^{24}$. Cet hebdomadaire conçu suivant la formule des grandes revues illustrées européennes finit par sacrifier le public élargi à la partisannerie politique et disparaît en 1883. De même, Le Monde illustré (Montréal, 1884-1902), qui devient L'Album universel (19021907), calque la manière du Monde illustré publié à Paris depuis 1857. Offrant nouvelles, anecdotes, feuilletons, poèmes, jeux de société, mode, cuisine, annonces, on y reconnait un cas type de revue populaire illustrée à la française ${ }^{25}$. Jusqu'à la fin du siècle, les périodiques qui cherchent à élargir leur audience vers le grand public expérimentent essentiellement dans une dépendance culturelle postcoloniale des formules métropolitaines, françaises surtout, mais aussi britanniques, tant en ce qui concerne les titres et la mise en page que les rubriques.

Le vent souffle d'ailleurs avec La Revue populaire (1907-1963), celle qui prétend faire concurrence à « tout autre magazine américain de même prix ${ }^{26}$ ». Avec une périodicité mensuelle, puis bimensuelle, offrant de 96 à 148 pages, elle diffuse les chroniques habituelles, de l'information de type encyclopédique, un long roman-feuilleton, des illustrations, suivant la formule américaine du magazine grand public $^{27}$. À peu de choses près, à partir de 1919, La Revue moderne lui fait concurrence sur le même terrain.

Traitons rapidement d'une première caractéristique, la question du support matériel, pour constater que ces périodiques du début du siècle peinent à imiter leurs modèles américains. Si le format et

${ }^{23}$ Le seul tirage connu, 1250 exemplaires, est celui de la dernière année, 1895. Il vient de The Canadian Newspaper directory, Montréal, A. McKim, 1892-1923.

${ }^{24}$ Voir Maurice Lemire et Denis Saint-Jacques (dir.), La vie littéraire au Québec, t. IV : 1870-1894: "Je me souviens ", Québec, Les Presses de l'Université Laval, 1999, p. 192-193.

25 Ibid., p. 194-195.

${ }^{26}$ La Revue populaire, Montréal, Cie de Publication Alpha, vol. 1, no 1 (décembre, 1907).

27 Lemire et Saint-Jacques, La vie littéraire au Québec, t. IV : 1870-1894, p. 194-195. 
la page de couverture s'ajustent facilement aux standards pratiqués, le manque de ressources ne permet d'abord que peu d'illustrations en couleurs, il n'y en a souvent que pour la seule couverture et une ou deux publicités, mais surtout cette publicité n'envahit d'abord pas autant l'espace rédactionnel. En un mot, ces revues apparaissent comme des parents pauvres de leurs grands frères d'outre-frontière. Toutefois, les différences les plus marquantes tiennent justement à cette modestie des moyens engagés, non à l'esprit, comme nous le verrons tout de suite. Au reste, vers les années 1960, le rattrapage se terminera : difficile alors de distinguer du point de vue du support matériel ce qui se fait ici de ce qui se fait là-bas.

Une deuxième caractéristique du magazine américain que reprend le magazine canadien-français, qui fait rimer publicité avec nationalité, représente ce qui intéresse les uns, les producteurs, et scandalise les autres, les intellectuels. L'idée même d'arrimer l'unité nationale à une démarche publicitaire suivant l'exemple américain a de quoi choquer la pensée conservatrice, qui s'emploie, au moment même de l'entrée des magazines sur le marché canadien-français durant le premier tiers du XX $x^{\mathrm{e}}$ siècle, à " restaurer » cette identité en péril dans divers périodiques. Elle s'y emploie, entre autres, dans des quotidiens comme L'Action sociale de l'archevêché de Québec, Le Droit des pères oblats à Ottawa ou Le Devoir d'Henri Bourassa à Montréal. Un périodique intellectuel, L'Action française ${ }^{28}$ dirigé par Lionel Groulx, vise l'ensemble des francophones d'Amérique et l'élite nationaliste s'y veut à l'écoute de "Notre maître, le passée ${ }^{29}$ ". Un catholicisme militant y réagit contre les dangers du consumérisme envahissant propagé par la presse moderne, tant journal d'information que magazine et, à voir l'ardeur des troupes réunies par ceux qui entendent « l'appel de la race $^{30}$ ", on ne donnerait pas cher de la peau des nouveaux périodiques capitalistes. Pourtant, La Presse, quotidien libéral, réalise

28 L'Action française, Montréal, Ligue des droits du français (1917-1927).

29 Lionel Groulx, Notre maître, le passé, Montréal, L’Action française, 1924.

30 Alonier de Lestres, L'appel de la race : roman, Montréal, Bibliothèque de l'Action française, 1922. 
en ces mêmes années une percée spectaculaire, atteignant les 121085 exemplaires par tirage dès avant la Première Guerre mondiale ${ }^{31}$.

Toutefois, pas plus que n'y arrivent pour leur part les quotidiens américains, La Presse ne couvre l'ensemble du territoire national en cause, ne serait-ce que celui du Québec. On comprend donc les éditeurs de vouloir suivre la voie ouverte aux États-Unis. Or, au lieu de la rapide percée américaine, l'aventure canadienne-française du magazine apparait laborieuse et, malgré les prétentions de l'un ou de l'autre des deux périodiques principaux, La Revue moderne et $L a$ Revue populaire, à s'afficher en tant que "magazine national des Canadiens français ", leur distribution n'atteindra les 100000 exemplaires par tirage qu’à partir de la fin des années 1950. L'enjeu, on s'en doute, concerne en particulier la nationalité problématique du média. Au Canada, en ce demi-siècle, le pouvoir économique passe de la domination du capital britannique à celle du capital américain; il y a, de plus, une élite économique locale anglophone. De sorte qu'on trouve, dans les mêmes pages, de la publicité pour les Empress de la compagnie britannique Cunard, à côté de celle pour les Ford américaines ou pour la Dow canadienne-anglaise, et même à côté de celle pour le magasin canadien-français Dupuis frères.

On ne doit pas se cacher la prééminence de la part anglophone dans cet ensemble; les francophones peuvent bien y trouver de l'information sur des produits intéressants à acquérir, mais ces produits se présentant souvent sous des noms anglais - indices d'une origine étrangère - ils ne peuvent aisément y déceler les contours clairs de leur identité canadienne-française. N'oublions pas, de surcroît, que le catholicisme militant des nationalistes défend, pour sa part, une identité rurale à l'opposé de celle, urbaine, du magazine. On sait que les almanachs, qui, avec leur calendrier des saisons, visent un public populaire, surtout campagnard, et tendent à disparaître ailleurs en

31 Beaulieu, Hamelin et al., La presse québécoise des origines à nos jours, t. III : 18801895. Voir aussi Jean de Bonville, La presse québécoise de 1884 à 1914 : genèse d’un média de masse, Québec, Les Presses de l'Université Laval, 1988. 
pays industrialisés, se maintiennent au Québec malgré la montée des quotidiens d'information et des magazines. L'Almanach du peuple de la librairie Beauchemin atteint un tirage de plus de 200000 exemplaires durant cette période ${ }^{32}$. On aura également noté, par ailleurs, l'exceptionnelle fortune, aussi tard que durant les années 1940 et 1950, du Bulletin des agriculteurs ${ }^{33}$. En ces années, ce dernier ajoute justement à son contenu informatif agronomique une section féminine comportant les rubriques habituelles, mode, nourriture, jeux, feuilleton et publicité, rapprochant ainsi l'identité rurale de celle, urbaine, axée sur la consommation des produits industriels. Il faut comprendre qu'il ne se trouve pas au Québec, même de façon imaginaire, une classe moyenne comparable à celle qu'invente et courtise alors le magazine américain. Les élites francophones, clergé, juristes, médecins et personnel politique, tendent à se voir en porteparole d'une identité commune canadienne-française fondée sur l'enracinement agricole. Encore durant les années 1950, le gouvernement de Maurice Duplessis défendra cette vision.

Pour rendre encore plus délicate la question de l'ancrage d'une identité stable, on constate que le pouvoir politique se situe à la fois à Ottawa et à Québec; c'est de ce pouvoir politique que devraient provenir les impulsions qui favoriseraient la convergence identitaire. Or ce pouvoir se trouve singulièrement partagé en ces années où les deux guerres mondiales entraînent des tensions fortes entre les deux groupes ethniques majoritaires, sans compter les divisions idéologiques entre les générations et les nouveaux mouvements politiques engendrés à l'occasion de la grande crise de 1929. On peut comprendre que ces confrontations rendent difficile la construction d'une identité cohérente, même pendant la Seconde Guerre mondiale, alors que la propagande de guerre fait converger, dans les magazines, à la fois la publicité et l'action gouvernementale fédérale.

\footnotetext{
32 Frédéric Brisson, «L'almanach au Canada français », dans Carole Gerson et Jacques Michon (dir.), Histoire du livre et de l'imprimé, vol. III : 1918 à 1980, p. 267.

33 Voir la note 11.
} 
Malgré le vote anti-conscription des Québécois, ces années et celles qui les suivent immédiatement marquent justement le tournant où les magazines, qui soutiennent l'effort de guerre, obtiennent enfin une circulation qui leur permet de prétendre à une représentativité nationale ${ }^{34}$. On peut, de plus, constater que l'action identitaire de construction nationale canadienne-française entreprise par les magazines tend à coïncider avec celle de la radio, employée à soutenir le même engagement. On peut juger que, comme pour la modernisation de la facture matérielle, cela arrive un peu tard par rapport à ce qui se produit au Canada anglais, dès les lendemains de la Première Guerre mondiale ${ }^{35}$, mais l'effort en ce sens ne s'en révèle que plus clair. C'est celui du nationalisme libéral pancanadien, celui de ce que l'on a appelé " l'idéologie du rattrapage ${ }^{36}$ ", celui déjà de la Révolution tranquille. Ces remarques font voir que les éditeurs montréalais n’ont pas uniquement emprunté la présentation du magazine américain, mais ont aussi poursuivi le même projet d'unification idéologique de leur public cible. Ils y ont œuvré non sans difficulté, et sans y parvenir entièrement.

\section{Spécificité du magazine canadien-français}

Mais il y a plus curieux, nous en avions montré un aspect à l'occasion d'un colloque sur l'année culturelle $1937^{37}$ : c'est la démarche qui invente, en liant fiction et publicité, la consommatrice moderne ${ }^{38}$.

${ }^{34}$ La Revue populaire passe de 40532 en 1941 à 82667 en 1956, selon Beaulieu, Hamelin et al., La presse québécoise des origines à nos jours, t. IV, p. 266-269; La Revue moderne passe de 31343 en 1940 à 97067 en 1956, toujours selon Beaulieu, Hamelin et al., La presse québécoise des origines à nos jours, t. V, p. 294-295.

35 Sutherland, The Monthly Epic: A History of Canadian magazines, p. 156-157.

36 Marcel Rioux, "Sur l'évolution des idéologies au Québec ", Revue de l'Institut de sociologie, Bruxelles, Université libre de Bruxelles, no 1 (1968), p. 95-124.

37 Ce colloque s'est tenu les 7, 8 et 9 novembre 2007 à l'initiative de l'équipe Penser l'histoire de la vie culturelle (PHVC), du CRILCQ et de la Chaire de recherche James McGill sur l'histoire comparée du Québec. Les communications présentées ont fait l'objet d'une publication : voir Lamonde et Saint-Jacques (dir.), 1937 : un tournant culturel.

38 Saint-Jacques et des Rivières, "Notre américanisation ", p. 154. 
Les magazines de ce temps présentent trois types principaux de contenus : articles d'information, réclames et récits de fiction. L'information est d'abord culturelle et politique (actualité littéraire, art, histoire, géographie, tourisme, politique canadienne); suivent la section féminine (mode, couture, cuisine, étiquette, soins de toutes sortes), le coin des enfants et le courrier des lecteurs. La publicité de marque présente des médicaments, sous forme de pilules ou de toniques, des savons et crèmes de beauté, de la nourriture et des boissons, des patrons qui suivent la mode, autant d'articles qui visent surtout la consommatrice; enfin, pour le couple, les voyages et l'automobile. La partie littéraire célèbre encore la poésie, mais surtout les grands romans complets «qui intéressent passionnément les lectrices qui aiment les beaux livres d'amour qui finissent par un mariage ${ }^{39}$ ". La fiction occupe la moitié de l'espace du magazine. Construite sur un canevas relativement stable, elle met en scène une jeune fille de bonne famille, mais sans moyens, qui fait la connaissance d'un homme beau et riche dont elle s'éprend. L'engagement est d'abord retardé par quelque empêchement. Cependant, la vertu de l'héroïne vainc les obstacles et les protagonistes se marient. Les romans de Delly, pseudonyme des Français Jeanne-Marie et Frédéric Petitjean de la Rosière, sont le prototype de ces romans d'amour catholiques que l'on retrouve dans les magazines. Nous considérons ce modèle comme une sorte de piège, un "piège à mariage " moderne. Le personnage de la jeune fille passe ainsi de l'intimité du rêve à l'engagement social par la formation d'une nouvelle famille. Parallèlement, la publicité du magazine capte l'attention de la lectrice, un peu comme si elle était une proie, et l'attire sur la place publique par le miroitement de produits, de beauté surtout, qui la rendent désirable sur le marché de la séduction. Les textes et les images de la publicité rejoignent ou même complètent l'histoire amorcée dans la fiction. Particulièrement présente dans les années 1940, cette publicité raconte que l'héroïne qui utilise tel savon pour

39 La Revue moderne, juillet 1927, p. 5. 
le visage sera admirée pour son teint. Un bel homme la choisira et elle saura conserver sa beauté grâce au savon ou à la crème. C'est le cas de la débutante Woodbury ${ }^{40}$ ! Les autres articles, détergents ou appareils électriques, épargneront à cette maîtresse de maison temps et fatigue par la suite. Car si la jeune épouse a les mains brisées par le labeur, le mari peut s'éloigner... La menace, à peine voilée, prend place sur les photos et dans le texte de la publicité de la crème Jergens : les mains rugueuses sont " contre l'amour » et les mains douces, "pour l'amour »!

À la différence des quotidiens, qui mettent de l'avant l'information politique, économique ou sportive, ou de leurs suppléments hebdomadaires qui offrent des rubriques sur la programmation théâtrale, radiophonique ou cinématographique, les magazines mensuels se consacrent à la synthèse, au commentaire et à l'information large à propos des arts, des lettres, des sciences et de l'histoire. La rédaction y évite la controverse, recherche le consensus qui assure l'étendue du public et, surtout, s'abstient de se mêler aux débats partisans de la politique. Enfin, la section féminine va jusqu'à marier les articles d'information aux renseignements fournis par la publicité au point qu'il est difficile, par exemple, de distinguer une chronique d'une publicité de mode.

Si cette conception du magazine trouve son origine aux ÉtatsUnis, elle tend à s'étendre un peu partout en Occident et on pourrait imaginer qu'il en va de même pour celle de la fiction. Or il semble bien que les choses se passent autrement au Canada français. Rien d'américain là, rien d'anglais, rien même de canadien; sauf rares exceptions, les éditeurs repiquent de l'édition française la très grande majorité des fictions qu'ils publient, puisant dans le corpus du roman d'amour catholique qui exalte cette France aristocratique à laquelle

${ }^{40}$ La débutante Woodbury est une héroïne qui revient souvent dans les publicités de cette compagnie de savon pour le visage et les mains. Grâce à l'emploi du savon Woodbury, la débutante sera belle, elle sera choisie par un prestigieux aviateur et se mariera. Voir notamment la publicité « Piste : une autre débutante Woodbury se marie ", La Revue populaire, novembre 1946, p. 21. 
la tradition conservatrice canadienne-française veut alors se rattacher. Ces textes français ne sont nullement adaptés, et marquis franccomtois, industriels lorrains, châteaux médiévaux, théâtres parisiens et montagnes des Alpes se succèdent sans qu'avocats montréalais, villages des Laurentides ou carnaval des neiges n'en viennent troubler l'atmosphère. Si le magazine est " américain ", les témoins s'entendent là-dessus, son imaginaire se compose en bonne part de fiction sentimentale française, la même exactement que l'on peut retrouver à l'époque dans les feuilletons du Devoir, par exemple. C'est dans ces romans d'amour que Bruchési, qui en avait publié dans La Revue moderne, aurait pu trouver, non un " reflet du véritable esprit français ", mais cet esprit même.

\section{Conclusion}

Nous pouvons conclure que non seulement l'aspect matériel du magazine canadien-français tend à se conformer à l'exemple américain, mais il manifeste aussi un souci idéologique de développer un public national axé sur la consommation féminine. En ce sens, le plus manifeste, Poirier et Bessette, les éditeurs, Carignan, l'écrivain, et Bruchési, le rédacteur, ne se trompent pas dans leurs déclarations convergentes. Pourtant, le ressort fondamental qui permet de constituer ce public (la fiction, le roman d'amour) reste, pour sa part, essentiellement français. Et aucun des derniers acteurs évoqués ne paraît le remarquer, tant ce caractère s'impose et est attendu. Cet agencement structurel rend le média que nous examinons ici tout à fait spécifique, et l'on pourrait décrire le public qu'il vise en ces termes : une consommatrice américanisée pour la vie matérielle, mais française d'esprit et, faut-il le dire, de cour.

Une telle conclusion reste pourtant déterminée par les présupposés dont elle procède, et l'on peut s'interroger sur le sens précis des dénotations nationalitaires à partir desquelles elle se construit. Il est improbable que " américain " veuille dire la même chose pour les trois témoins (éditeur, écrivain et directeur de publication) dont nous 
avons rapporté les propos. En fonction de leur position dans le champ médiatique, on peut supposer que les premiers vantent le caractère " moderne ", « dernier cri » du produit qu'ils mettent en marché. Le deuxième, auteur en quête de débouché, se trouve déçu des publications qui n'accordent pas de place à la création littéraire et à l'information intellectuelle qu'on trouve alors dans les pages du Mercure de France, de La Nouvelle Revue française ou, même, de la fort bourgeoise Revue des deux mondes. Le troisième, nationaliste, ne peut manquer d'être contrarié après avoir échoué à rapprocher La Revue moderne de l'idéologie qu'il veut soutenir; le travers qu'il déplore doit lui apparaître comme relevant de l'urbanité croissante et ces publications doivent manquer à ses yeux d'engagement politique.

Cependant, même si l'on admet que ces acteurs sociaux s'accordent jusqu'à un certain point sur l'américanité des magazines canadiens-français de leur époque, il est facile de constater que ce modèle se diffuse à une telle vitesse en Grande-Bretagne, en France ou au Canada anglais que, dès la deuxième moitié du $\mathrm{xx}^{\mathrm{e}}$ siècle, il joue un rôle beaucoup moins important dans la construction des identités nationales. Si l'on y regarde de près, les articles d'information générale, de mode, de santé, de tenue de maison et autres ne diffèrent pas fondamentalement d'un marché à l'autre. Tous les magazines "familiaux " ou "féminins " de l'époque offrent de la fiction, essentiellement sentimentale. On sait enfin que la publicité envahit la presse dès l'apparition de la presse quotidienne grand public en France et en Grande-Bretagne. Le cas américain du magazine n'en présente qu'une radicalisation plus éclatante que d'autres. Les éditeurs américains ont un moment synthétisé des apports provenant de Grande-Bretagne et de France avec ceux qui leur étaient propres et relancé ainsi un mouvement qui a pu mobiliser par opportunisme une tendance nationalitaire. C'est bien cette homogénéité relative des traits constitutifs du média qui lui permet si aisément de franchir les barrières linguistiques et culturelles de marchés de plus en plus perméables. Pour les producteurs et le public du temps, «américain " dénote ce qu'on appelle aujourd'hui " capitaliste consumériste ». 
Comme bien des recettes, le magazine perd ses caractéristiques locales en voyageant. Et, à la différence du cinéma grand public hollywoodien qui nous provient toujours des États-Unis, le magazine contemporain, comme celui de la première moitié du $\mathrm{xx}^{\mathrm{e}}$ siècle, émane de partout, car il est de partout.

Nous pourrions ajouter une dernière observation. Lorsqu'on analyse les échanges interculturels, il ne faut pas oublier, d'une part, qu'ils se produisent par l'importation d'objets, de formes ou d'idées venus d'ailleurs. En ce sens, on parle d'influences. D'autre part, ces échanges se réalisent dans des conditions particulières à leur milieu de réception, conditions qui les transforment et leur donnent de nouvelles valeurs. En cet autre sens, on parle d'adaptation. Si les contemporains de ces changements persistent à les référer au lieu dont ils proviennent, ils le font rarement dans un souci objectif de compréhension. Le caractère étranger, en particulier, reste fréquemment connoté de valeurs positives ou négatives fortes. Ici, par exemple, " américain " veut dire déchéance pour les uns et progrès pour les autres; son sens est d'abord polémique. 